Università degli Studi di Genova

DISEFIN - Series of Economic Working Papers

16126 Genova - via vivaldi 5 - Fax +39010 2095223

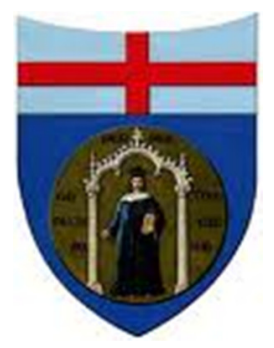

Shifting the risk in pricing and reimbursement schemes? A model of risk-sharing agreements for innovative drugs

Stefano Capri - Rosella Levaggi

wp n. 2/2011

MAY 2011 
"DISEFIN Working Papers on line"

series of economic working papers

published online by

Research Doctorate in

Economics and Finance of European Union

Ph.D School in New technologies and Social Sciences

University of Genoa

Founder:

Amedeo Fossati

Editor-in-Chief:

Marcello Montefiori

Editorial Board:

Paul De Grawe

Francesco Figari

Amedeo Fossati

Luca Gandullia

Eric Gaspérini

Andrea Monticini

Carlo Perroni

Web site:

http://www.disefin.unige.it 


\title{
Shifting the risk in pricing and reimbursement schemes? A model of risk-sharing agreements for innovative drugs
}

\author{
Stefano Capri \\ Institute of Economics, \\ Cattaneo - LIUC University, \\ Italy
}

\author{
Rosella Levaggi \\ Department of Economics, \\ University of Brescia, \\ Italy
}

\begin{abstract}
Risk sharing is becoming an increasingly popular instrument to regulate the price of new drugs. In the recent past, forms of risksharing agreements between the public regulator and the industry have been proposed and implemented, but their effects on price and profits are still controversial. in this paper we propose a model aimed at studying the effects on price and expected profit of several risksharing agreements between a regulator and the industry, based on the ex post effectiveness of the drug (i.e. the efficacy resulted in the real medical practice). We assume that the probability of being listed (approved and reimbursed) depends on the relative performance of the new drug in terms of effectiveness and budget required. The price is set according to the declared efficacy of the new drug, but if ex post the effectiveness falls short of what declared, several forms of penalties may be used by the regulator. We show that the number of patients that are treated is not necessarily affected by risk-sharing/risk-shifting mechanisms; the price for which the drug is listed may be higher than without risk-sharing, but the expected profit of the industry is: a) always lower for risk-shifting schemes; b) for true risk-sharing it depends on the bargaining power of the company. This result is however valid only if the listing process is not affected by risk sharing. If this is not the case, risk sharing mechanisms may increase the expected profit of the industry.
\end{abstract}


Keywords: Drug pricing, Risk-sharing, Efficacy, Effectiveness

Jel Classification: I11, I18, D45 


\section{Introduction}

The rapid increase in pharmaceutical expenditure and the need to control the health care budget has sparked renewed interest in drug pricing policies (Claxton, 2007). Value based schemes and risk sharing agreements have been proposed (De Pourville, 2006, Cook et al, 2008; Adamsky et al, 2010) in order to curb expenditure and to improve the value for money of health care expenditure.

Rising costs are a challenge to healthcare policy makers because high prices put budgetary pressure on governments that try to maintain access to drugs for the population at an affordable cost. ${ }^{1}$ For this reason, most EU member states control the prices of reimbursable medicines.

The aim of these regulatory mechanisms is to find an optimal trade-off between the need to incentivate $R \& D$, consumers'protection and to secure value for money in the use of public funds. The market for pharmaceuticals is not, for various reasons, a competitive market. ${ }^{2}$ The demand side is characterized by uncertainty on the effectiveness of the drug and patients'inability to translate their need for improved health into the demand for a specific treatment. The agency relationship between the patient and the physician ${ }^{3}$ means that that choice of the drug is largely entrusted to the latter, with a possible distortion of the market. The regulations and restrictions laid down by Government agencies in this sector are themselves a source of distortion. Pharmaceutical price regulation methods in non-US markets are heterogeneous and include, for example, direct price regulation through a negotiation process (e.g. France and Italy) and indirect price regulation through limits on reimbursement under social

\footnotetext{
${ }^{1}$ In Europe, about $75 \%$ of pharmaceutical expenditure is reimbursed from public funds (OECD, 2009)

${ }^{2}$ See Zweifel, Breyer and Kifmann (2009) chap.12 and Capri and Levaggi (2006)

${ }^{3}$ See Barigozzi and Levaggi (2008) and references therein
} 
insurance programmes (e.g. Germany and Japan) (Danzon and Chao, 2000b; Capri and Levaggi, 2002; 2006).

One of the most innovative methods applied in pricing schemes is represented by risk-sharing agreements. In the pharmaceutical market risk sharing is said to occur when the risk involved (in this case the cost of a particular drug therapy) is shifted from one stakeholder to another, from the government to the industry and vice versa, in order to alleviate some of the concerns about uncertainty. Adamsky et al. (2010) review the principal agreements in this area and argue that are two types of risk sharing: performance-based and financial based contracts. Performance-based contracts focus on the effectiveness of the new drug, whereas risk-sharing financial-based agreements involve expenditure considerations.

Towse and Garrison (2010) show that, from a pure economic point of view, most of these schemes should in fact be classified as risk shifting agreements since they are in fact designed to reduce the price paid for the drug. The industry may prefer this scheme to a straightforward price reduction for several reasons. In some markets the pharmaceutical company has high incentive in showing similar prices respect to other countries -ex-factory prices- in order to assure an homogeneous pricing policy. Therefore they are willing to accept the imposition of "undisclosed" discounts/rebates wich are part of the no trasnsparent risk-sharing agreement. The final result of the scheme (i.e. whether it is risk sharing or risk shifting) is mainly determined by the negotiation rules the regulator foresees. If, as it seems to happen in several countries, risk sharing is used to increase the probability of listing new drugs, the effect of risk sharing agreements are not so clearcut as the present literature seems to suggest.

The aim of this paper is to study the effects of risk-sharing in the market for new, innovative drugs. We will study the introduction of these schemes under different rules as concerns the negotiation process and risk sharing arrangements itself. We show 
that in general these contracts may increase the price paid for the new drug, but the expected profit for the pharmaceutical industry is always lower under these schemes. In other words, they should be classified as risk shifting. This result holds only if the listing process is independent of the contract, i.e. the probability of being listed is not affected by the risk sharing arrangement. If this is not the case, the contract may involve true risk sharing between the parties and it may considerably increase the profit of the regulated industry.

The article will be organised as follows: in the following section we explain the concept of risk sharing in the pricing scheme for new drugs, in section 3; in section 4 and 5 we present our pricing scheme with and without risk-sharing respectively; in section 6 we show how our pricing mechanism can be used to represent most of the systems used by regulators; in section 7 we show the difference between a risk-sharing and a risk-shifting scheme and argue why a company may still prefer to be listed with risk-shifting. Finally in section 8 the main conclusions of our paper are drawn.

\section{Risk-sharing concepts}

In most countries where healthcare is mainly financed through public expenditure the price of new drugs is strictly controlled by government agencies. The control is made through the listing process, a detailed procedure that allows new drugs to be commercialised and possibly to be reimbursed.

The listing process is country-specific, but its essential features can be summarised as follows: the outcome is uncertain (the effect of the drug on the patient's health), and the probability of success depends on several factors such as its cost effectiveness (usually measured through its Incremental Cost Effectiveness Ratio - ICER ) and the expected budget required to take care of the potential patients that will benefit from the new drug. 
This process is highly uncertain because the drug becomes an input of a more complex process whose outcome is patient's health gain. This process depends on the quality of the drug itself, but also on patient's ability to regain health and this element is very difficult to be foreseen. For this reason the productivity of health care treatments is measured by its efficacy and its effectiveness.

The efficacy is the theoretical improvement in health that can be obtained through health care. For drugs efficacy is proxied by the health improvements obtained through a randomised clinical trial where the latter are measured within a controlled experiment. The effectiveness is the actual improvement in health care that a large number of patients will gain from using that treatment into the real medical practice. Ideally the effectiveness of a new drug should be very close to its efficacy; in practice effectiveness is lower and the gap may be quite large. Several are the causes for such discrepancy: the role of patients' compliance, the interactions with other drugs when patients have several pathologies and the appropriateness of physicians' prescription behaviour. This is a very important problem for the regulator because it has to pay for something that may prove to be much less effective than what expected.

The expected efficacy of the drug increases the probability of being listed and, possibly, of obtaining a high price. The number of patients have a countervailing effect on expected profits since it reduce the probability of being listed (higher budget required), but it increase profits if the outcome of the listing process is positive.

Once the drug has been introduced, almost all regulatory systems require greater effort in the post-marketing monitoring of drugs, not only from a purely medical perspective (DePouvourville, 2006). Public authorities, in fact, schedule procedures to control the drug for possible side effects. Until recently only few regulators had verified the real ex post value for money of the drug. This failure to verify efficacy and volume ex post creates perverse effects on the regulatory system. The industry, in fact, may have an 
interest in overestimating the efficacy and underestimating the number of people that will benefit from the drug in the listing process, given that both parameters will not be controlled by the regulator ex post.

It is very difficult to verify the discrepancy between efficacy and effectiveness, apart from for very targeted drugs such as cancer drugs. This is because the number of patients is limited and already controlled. In Italy, for instance, a specific registry for expensive cancer drugs has been created and for some of these drugs a variety of risk-sharing scheme is applied. ${ }^{4}$ The patient is registered in the website and treatment is initially paid by the Italian NHS. If treatment fails (progressive disease or unacceptable toxicity at or before the agreed time), the pharmaceutical company reimburses (money or corresponding amount of drug) the whole delivered treatment or $50 \%$ of it. An example for the UK is Janssen-Cilag's Velcade (bortezomib, see NICE, 2007) in which patients who demonstrate a $50 \%$ response rate at first relapse are eligible to continue treatment on the NHS, otherwise the manufacturer refunds the NHS.

A new strand of literature is developing to study risksharing. Zaric and O'Brien (2005) study the effects of rebates in the pricing mechanism when the quantity sold exceeds the negotiated quantity. As for the performance of the drug, Barros (2010) studies the welfare properties of specific risk-sharing mechanisms while Zaric and Xie (2009) examine two different risk-sharing agreements in an intertemporal setting. In their model the company applies for listing, proposes a price and sets a marketing strategy to sell the drug. The efficacy of the latter is a random variable and a penalty is imposed on the firm if its effectiveness falls below a specific threshold. The penalty may consist in delisting in the second period or in a rebate. The authors show that there is not a superior instrument: the choice depends on the environment

\footnotetext{
${ }^{4}$ See http://antineoplastici.agenziafarmaco.it/
} 
(measured by uncertainty and consumers preferences) and the objectives pursued by the regulator.

In this article we propose a general modelling framework that allows study of the risk-sharing properties of several pricing schemes, their effects on the number of patients treated and on the expected profit of the company selling the drug. Our framework allows for differentiation between risk-sharing and risk-shifting schemes and has important policy implications: the number of patients that are treated is not necessarily affected by risksharing/risk-shifting unless the probability of being listed changes because of risk shairng ; the price for which the drug is listed may be higher than without risk-sharing, but the expected profit of the industry is: always lower.

\section{The model}

In this paper we want to model the pricing strategy of an industry which is about to ask listing in market where other drugs already exists to treat a specific condition. To simpliy matters, we assume that in the market we analyse, patients are treated using a drug that is currently listed and has the following characteristics:

- the efficacy is equal to $L / 2$ this value is obtained by the randomised clincal trials that the industry has to produce to the regulator in order to obtain the approval of the drug. However the effectiveness $E^{\circ}$ of the drug is not known. We assume that $E^{\circ}$ may lye in a range of values $(0, L)$ with a uniform probability;

- the drug is listed for a price equal to $p^{\circ}$ and a quantity equal to $x^{\circ}$ : As a consequence, the public expected expenditure for this drug is equal to $B^{\circ}=p^{\circ} x^{\circ}$;

- the regulatory process by which the drug has been approved and listed is the same as the one that will be used for the new drug. In other words we assume a long run equilibrium where drugs currently on the market are priced on the basis 
of their effectiveness. This assumption is in line with the most recent literature on value based drug price (Claxton, 2008)

In this market, a new drug is about to be marketed to treat the same condition. Its effectiveness $E$ is not known at this stage; what it can be observed is the expected efficacy $E D$ which we assume to be equal to $A / 2$. As for the drug already on the market, $t$ is derived from the randomised clinical trials the firm has to carry out before the drug is approved, hence it is observable by the industry and the regulator. The industry knows that the effectivenes of the new drugs lies within a range of values $(0 ; A)$ with a known probability distribution $g(E)$ with $G(0)=0 ; G(A)=1$ : To simplify the exposition, we assume that the distribution for this function is uniform, i.e. $g(E)=1 / A$. The marginal cost $\mathrm{c}$ to produce the drug is approximated to zero, and the firm has to incur a fixed cost $F$ for R\&D investment that is sunk at the time of listing. To simplify matters, we assume that one unit of the drug is sufficient to treat one patient so that the number of doses and the number of patients are equivalent.

\subsection{The listing process}

The outcome of the listing process (i.e. the approved price, the level of reimbursement, the limitations) is uncertain and depends on the effectiveness of the new drug, the price $(p)$ and the number of patients to be treated $(x)$.

The schemes that are usually used for listing uses $E D$ as a proxy for the effectiveness of the new drug. The rationale for this choice is that this value can be observed by the industry and the regulator at the time of listing. However, this choice appears to be more and more unsatisfactory: the price of new drugs is usually higher than existing active principles, the difference between expected and true, ex-post effectiveness is sometime quite large. 
For these reasons "risk sharing schemes" are used. Several forms have been adopted, but they all share a common principle: the industry engages in supplying a minimum level of effectiveness verifiable ex post. If the latter fall short of what promised, the industry repays the regulator according to specific contractual rules, as a sort of "money back guarantee".

The price $p$ and the number of patients to be treated, $x$; are the result of a bargaining between the company and the regulator where the price and the quantity may be decided by the firm, by the regulator or by the market depending on the regulatory framework (Capri and Levaggi, 2006; Jelovac, 2003; Claxton, 2007).

To show the effects of risk sharing, we assume that the price of the drug depends on three parameters:

- $\alpha$, a parameter set by the regulator. It may be interpreted as society's willingness to pay for health improvements (measured in terms of incremental effectiveness) in a class of specific treatments. In Italy this parameter may vary from treatment to treatment and its setting is not transparent, but this choice represents almost an exception. Most countries are nowadays adopting the UK system of NICE (National Institute for Clinical Excellence) thresholds that define the maximum willigness to pay for a specific class of treatments (Appleby et al, 2000; KCE, 2008, McCabe, 2008)

- $D$ the level of efficacy the industry defines for the money back guarantee; it must lie in the range $(0 ; A)$ but it may not necessarily be equal to its expected value, $A / 2$. This is because the firm may be more or less optimistic about the success of the drug in treating patients outside the randomised clinical trial;

- $s D$; a specific threshold for assessing whether the industry receives the full price with $0 \leq s \leq 1$ : For $s=0$ no risk sharing is foreseen while values of $s=1$ the price of the drug is paid only if the effectiveness is at least equal to the target level. 
- $k$; a rebate on the price if the ex post effectiveness $(E)$ is lower than $D$ with $0 \leq k \leq 1$. For $k=1$ the payback is equal to the price paid to the company.

In other words, the agreement between the pharmaceutical company and the regulator takes this form:

$$
\begin{array}{lll}
p=\alpha D & \text { if } & E \geq s D \\
p=\alpha D(1-k) & \text { if } & E<s D
\end{array}
$$

and the expected price the company receives is equal to:

$E(p)=\alpha D(1-k) \int_{0}^{s D} \frac{1}{A} d E$

This risk-sharing mechanism is very general and for specific set of parameter it reproduces similar to the one proposed by Zaric et al. (2009) and by Barros (2010). It is interesting to note that all the risk sharing formulae are asymmetric: they foresee a rebate if ex post effectiveness is lower than what guaranteed, but they never foresee a premium if the drug performs better than expected.

The number of patients that will be treated is defined by $x$. We assume that this number is contractible and can be verified and that it has be stated by the company at the time of listing:The company applies for the drug to be reimbursed by the regulator. The latter may or may not decide to grant reimbursement; this decision depends on $D$ and on the budget $B=p x$ that is necessary to take care of the possible benefiter $(x)$ of the new drug. Both variables are measured in relation to the market in which the new drug is introduced.

In particular, we assume that the probability of being reimbursed can be defined by $v\left(\frac{D}{D^{\circ}}, \frac{B}{B^{\circ}}\right)$ where $D^{\circ}$ and $B^{\circ}$ is the efficacy of the drug and the budget spent for the active principle that is currently used to treat patients with the same condition. The 
probability of being listed is increasing in $D$ and decreasing in $p x^{5}$; as in Zaric and O'Brien (2005), the function is assumed to be separable and additive in $\frac{D}{D^{\circ}}$ and $\frac{B}{B^{\circ}}$, i.e.principle that is currently used to treat patients with the same condition. The probability of being listed is increasing in $\mathrm{D}$ and decreasing in px5; as in Zaric and O'Brien (2005), the function is assumed to be separable and additive in $\mathrm{D} D$ and $\mathrm{B} B$, i.e.

$$
v\left(\frac{D}{D^{\circ}}, \frac{B}{B^{\circ}}\right)=v_{1}\left(\frac{D}{D^{\circ}}\right)-v_{2}\left(\frac{B}{B^{\circ}}\right)
$$

To show the effects of risk sharing arrangements on prices, volumes and expected profits of the industry, in the following section we present a benckmark model where no risk sharing is foreseen.

\section{Listing process without risk-sharing}

In the absence of any other information, the pricing rule set by the regulator will depend on efficacy $\left(\left(D=\frac{A}{2}\right)\right)$, but the system does not foresee any penalty if ex post $E<\frac{A}{2}$, ; i.e. $s=k=0$. In this case the price $\left(p^{N R}\right)$ can be written as:

$$
p^{N R}=\alpha \frac{A}{2}
$$

If listed, the industry receives $\alpha \frac{A}{2}$ for each patient treated, independently of $E$, the effectiveness verified ex post. The only discretionary variable for the company is $x$; the number of patients, which will be determined through the maximisation of its expected profit:

$$
\operatorname{Max}_{x} E \Pi=\left(v_{1}\left(\frac{D}{D^{\circ}}\right)-v_{2}\left(\frac{B}{B^{\circ}}\right)\right) \alpha \frac{A}{2} x
$$

$$
\text { 5 i.e. } \frac{\partial \pi(E D ; p x)}{\partial E D}>0 \text { and } \frac{\partial^{2} \pi(E D ; p x)}{\partial E D^{2}} \leq 0 \text { and } \frac{\partial \pi(E D ; p x)}{\partial p x}<0 \text { and } \frac{\partial^{2} \pi(E D ; p x)}{\partial p x} \geq 0
$$


As in Zaric and O'Brien (2005) we assume that the marginal probability of being listed is linear; the F.O.C. can be written as:

$-\frac{1}{2} \alpha A^{2} \frac{\pi_{2} \alpha x L-\pi_{1} p^{\circ} x^{\circ}}{L p^{\circ} x^{\circ}}$

The optimal solution for $\mathrm{x}$ can be written as:

$x^{N R}=\frac{x^{\circ}}{2} \frac{\pi_{1}}{\pi_{2}}$

The price of the new drug is outside the control of the industry in the listing process, unless the industry can infuence the value of $A / 2$ through the randomised clinical trial. The number of patients that the industry will target for the new drug may be greater or smaller than for the drug already listed. It will depend on the parameters of the probability of listing set by the regulator. $\frac{v_{1}}{v_{2}}$ is in fact the ratio of the marginal effect on the probability of being listed deriving from an increase in the effectiveness and in the budget. $\left(\left(D=\frac{A}{2}\right)\right)$ This ratio may be controlled by the regulator. For example, if the latter wants the same number of patients treated with the old and the new drug $\left(x^{N R}=x^{\circ}\right)$, it will have to put greater weight on the efficacy compared to the budget $^{6}$. To some extent, this may not be a perverse effect of regulation given that the new drugs are more effective, but also more targeted on a restricted number of patients (Pirmohamed and Lewis 2004; Danzon and Towse, 2002).

Substituting equation (3) into (4) we can write the expected profit as:

$$
E \Pi^{N R}=\frac{1}{2} \frac{x^{\circ}}{L} \frac{v_{1}^{2}}{v_{2}} \alpha\left(\frac{A}{2}\right)^{2}
$$

\footnotetext{
${ }^{6}$ i.e. if $\pi^{1}$ (the marginal probability of being listed) is twice $\pi^{2}$ (the probability of being listed).
} 
The profit is increasing in $\alpha$ and in the expected efficacy of the new drug. The number of patients treated by the old drug $\left(x^{\circ}\right)$ increases the profit while its expected efficacy $\left(\frac{L}{2}\right)$ reduces it. This result is in line with what expected.

\subsection{A risk shifting mechanism}

Let us now assume that the regulator makes the industry share the risk that the effectiveness $E$ falls below $A / 2$ : In this case, the price is still set according to the rules presented in the previous section, but the industry has to pay a penalty if the effectiveness $E$ is lower than $A / 2$ : In terms of standard industrial economics this payment can be classified as pay for performances scheme which always induce risk shifting since it reduces the expected profit of the industry. In this new environment, the company chooses the number of patients that maximises the following function:

$\operatorname{Max}_{x} E \Pi=\left(v_{1}\left(\frac{A}{L}\right)-v_{2}\left(\frac{\alpha \frac{A}{2} x}{p^{\circ} x^{\circ}}\right)\right)\left(\alpha \frac{A}{2}(1-k) \int_{0}^{s \frac{A}{2}} \frac{1}{A} d E\right) x$

If the marginal probability of being listed is linear as in the previous example, the optimal level for $\mathrm{x}$ can be written as: ${ }^{7}$

$x^{S F}=\frac{x^{\circ}}{2} \frac{v_{1}}{v_{2}}$

By comparing equation (6) with equation (9) we can see that the number of patients which the company is applying to treat is the same, i.e. the risk-shifting formula does not alter the decision of the company as regards the number of patients for whom it is agreed the new treatment will be made available if listed.

Through substitution of the optimal quantities in the profit function we can determine the expected profit:

\footnotetext{
${ }^{7}$ See appendix A.2.1
} 
$E \Pi^{S F}=\frac{1}{2} \frac{x^{\circ}}{L} \frac{v_{1}^{2}}{v_{2}} \alpha\left(\frac{A}{2}\right)^{2}\left(1-\frac{1}{2} k s\right)$

The expected profit $E \pi^{S F}$ is lower than for no risk sharing case $\left(E \pi^{N R}\right)$. The two equations are in fact equal for $k s=0$; but profit is decreasing in $k s$ as might be expected.

\section{A risk-sharing mechanism?}

Risk sharing agreements are becoming popular for cancer drugs which are reimbursed to the firm only if they are effective by different combination of schemes: e.g. in Italy nilotinib and sorafenib are not reimbursed after the second cycle of therapy if the patient does not respond to the first cycle; avanstin is fully reimbursed only for respondent patients after 15 cycles; sprycell is reimbursed at $50 \%$ of the price if there is a progression of the disease after the first cycle. In this case a threshold for reimbursement is defined in relation to the level of declared efficacy. For the more general case, the price the company is expecting to receive is represented by equation (2).The company aims to maximise its expected profit and to do so it has to choose the value of $D$ and $x$ that maximises the following function ${ }^{8}$ :

$\operatorname{Max}_{D, x} E \Pi=\left(v_{1}\left(\frac{D}{D^{\circ}}\right)-v_{2}\left(\frac{\alpha D x}{p^{\circ} x^{\circ}}\right)\right)\left(\alpha D(1-k) \int_{0}^{s D} \frac{1}{A} d E\right) x$

As in the previous examples, if the marginal probability of being listed is linear, the optimal level for $D$ and $x$ can be written as ${ }^{9}$ :

\footnotetext{
${ }^{8}$ Given that during the negotiation process neither party knows the true eqectiveness, the price that the regulator uses in the decision to list the drug is $p=\alpha D$.

${ }^{9}$ See appendix A.3.1
} 
$D^{S H}=\frac{2}{3} \frac{A}{k s}$ if $k s>\frac{2}{3}$

$D^{S H}=A \quad$ if $k s<\frac{2}{3}$

$x^{S H}=\frac{x^{\circ}}{2} \frac{v_{1}}{v_{2}}$

The parameters $k$ and $s$ play a similar role in this model. From a policy point of view this means that the regulator may choose the payback level and the threshold independently, but it is the combination of these two parameters that determine the choice of the industry. It is interesting to note that the penalty should be quite severe to avoid the industry to declare a very high level of effectiveness of the drug. (allargare: esempio). In what follows we will consider only the internal solution, i.e. we assume that the penalty is sufficiently high for $D^{*}<A$. In this later case, through substitution of the optimal quantities in the profit function we can determine the expected profit:

$$
E \Pi^{S H}=\frac{1}{27} \frac{A^{2}}{k^{2} s^{2} D^{\circ}} \alpha \frac{v_{1}^{2}}{v_{2}} x^{\circ} \quad k s \geq \frac{2}{3}
$$

If the price of the old drug has been set according to the same rules, $D^{\circ}=\frac{2}{3} \frac{L}{k s}$ and the expected profit can be written as:

$$
E \Pi^{S H}=\frac{1}{18} \frac{A^{2}}{k s L} \alpha \frac{v_{1}^{2}}{v_{2}} x^{\circ} \quad k s \geq \frac{2}{3}
$$

This formula represents the profit both for the case in which only the effective therapies are paid for (in which case $k=1$ ) and the more general case of a rebate. In this latter case only $0 \leq s \leq 1$ is the parameter set by the regulatory authority. For $s=1$ the company is reimbursed only if the ex post effectiveness is at least equal to the declared efficacy. From equation (12) it is interesting to note that the quantity for which the company requests listing is the same as in the no risk-sharing case. In this case our model does not predict 
an increase in the number of cases treated, a possible problem that Barros (2010) points out for risk-sharing agreements. The difference in the results of the two models is explained by the presence in our approach of a listing process also for the risk sharing agreement. Such result has important policy implications: risk-sharing does not necessarily mean that more patients will be treated, unless the listing process is changed. This important conclusion will be made more explicit in the following paragraph where we present an alternative decision process as concerns risk sharing.

As in the previous case, the profit is increasing in . In this case it is also decreasing in $k s$, as might be expected.

\section{Listing process and risk sharing}

So far we have assumed that the listing process is unaffected by the risk sharingarrangements. In actual fact, and especially in the countries where negotiation process in not very transparent, it may well be the case that the negotiation process itself changes. In particular it may happen that the parameters of the listing process changes and that listing becomes a more likely outcome of the bargaining process. In terms of our model we can assume that, as a result of the introduction of risk sharing the probability distribution becomes $v^{\prime}\left(\frac{D}{D^{\circ}}, \frac{B}{B^{\circ}}\right)>v\left(\frac{D}{D^{\circ}}, \frac{B}{B^{\circ}}\right)$ so that the expected profit the company faces is equal to:

$\operatorname{Max}_{D, x} E \Pi=\left(v_{1}^{\prime}\left(\frac{D}{D^{\circ}}\right)-v_{2}^{\prime}\left(\frac{\alpha D x}{p^{\circ} x^{\circ}}\right)\right) \alpha D x+\left(\alpha D(1-k) \int_{0}^{s D} \frac{1}{A} d E\right) x$

The change in the listing process may be anticipated by the company or not. In what follows we examine both cases.

\subsection{Unanticipated change in the listing process}

The most discussed case by the literature as concerns unanticipated changes is represented by an environment where the the price is set according to the expected effectiveness of the drug 
and the firm sets the volume. A change to a regime where the firms is asked to share the risk with the regulator is a clear risk shifting agreement as shown in section 4.1.

However, this is true only if the system moves from the listing process described by equation (4) to (8). If the regulator, as a result of risk sharing changes the probability of being listed and makes listing more probable, the firm to get a profit according to equation (15), the gainers and losers of risk sharing may be different from what the current literature seem to point out. The optimal price and volume are still represented by equations (9) and (2), but the expected profit should be evaluated by substituting these equations in (15). If we assume that the marginal probability of being listed is linear and that $v_{1}^{\prime}=r_{1} v_{1}$ and $v_{2}^{\prime}=r_{2} v_{2}$ the expected profit can be written as:

$E \Pi^{S F_{N A}}=\frac{1}{8}\left(r_{1}-\frac{1}{2} r_{2}\right) \frac{x^{\circ}}{L} \frac{v_{1}^{2}}{v_{2}} \alpha A^{2}\left(1-\frac{1}{2} k s\right)$

for the "risk shifting" case and

$E \Pi^{S H_{N A}}=\frac{1}{27}\left(2 r_{1}-r_{2}\right) \frac{A^{2}}{k^{2} s^{2} D^{\circ}} \alpha \frac{v_{1}^{2}}{v_{2}} x^{\circ}$

for the risk sharing case.

For a listing process to be more generous we have to assume that $r_{1} \geq 1$ and $r_{2} \leq 1$ which means that the expected profit is higher than under the previous systems, as one might expect.

\subsection{Anticipated change in the listing process}

If the company correctly foresees the change in the listing process, the optimal $D$ and $x$ can be obtained by the maximisation of equation (15). In appendix A.2.2 we show that for the risk shifting case, the company sets:

$x^{S F_{A C}}=\frac{x^{\circ}}{2} \frac{v_{1} r_{1}}{v_{2} r_{2}}$ 
and the expected profit will be equal to:

$E \Pi^{S F_{A C}} \frac{1}{16} \frac{r_{1}^{2}}{r_{2}} \frac{x^{\circ}}{L} \frac{v_{1}^{2}}{v_{2}} \alpha A^{2}\left(1-\frac{1}{2} k s\right)$

For the risk sharing case, if we assume that the marginal probability of being listed is linear, the optimal level for $D$ and $x$ can be written as ${ }^{10}$ :

$$
\begin{aligned}
D^{S H_{A C}} & =\frac{2}{3} \frac{A}{k s} \text { if } k s>\frac{2}{3} \\
D^{S H_{A C}} & =A \quad \text { if } k s<\frac{2}{3} \\
x^{S H_{A C}} & =\frac{x^{\circ}}{2} \frac{v_{1} r_{1}}{v_{2} r_{2}}
\end{aligned}
$$

and the optimal profit (for $k s>\frac{2}{3}$ )) will be equal to:

$$
E \Pi^{S H_{A C}}=\frac{1}{18} \frac{r_{1}^{2}}{r_{2}} \frac{v_{1}^{2}}{v_{2}} \frac{x^{\circ}}{L} \alpha \frac{A^{2}}{k s}
$$

\section{Policy implications}

Who are the gainers and the losers of the risk-sharing mechanism proposed for the drug industry?

From a purely economic point of view, one of the most important arguments for not introducing risk-sharing mechanisms is that they may increase the price of the drugs, hence public expenditure. The company on the other hand may feel that the system is depressing expected profit. In our model, given the assumption of zero marginal production costs as regards the drug, we can simply compare the expected profit of the company under the two arrangements. In fact, given the assumption of zero marginal costs to produce the drug, public expenditure and

${ }^{10}$ The maximisation process is presented in appendix A.3.2 
expected profit in our model differ because of $F$ that in our model is assumed to be a fixed sunk cost that the industry has borne at the time it asks for listing and that is not going to be affected by this process. This imply that we can evaluate the impact on public expenditure of risk sharing mechanisms by simpy comparing the expected profit of the industry under the different settings. Table one summarises the results presented in the previous section.

\begin{tabular}{|llll|}
\hline & $p$ & $x$ & $E \Pi$ \\
Benchmark & $\alpha \frac{A}{2}$ & $\frac{x^{\circ}}{2} \frac{v_{1}}{v_{2}}$ & $\frac{1}{8} \frac{x^{\circ}}{L} \frac{v_{1}^{2}}{v_{2}} \alpha A^{2}$ \\
\hline Risk shifting (SF) & $\alpha \frac{A}{2}$ & $\frac{x^{\circ}}{2} \frac{v_{1}}{v_{2}}$ & $\frac{1}{8} \frac{x^{\circ}}{L} \frac{v_{1}^{2}}{v_{2}} \alpha A^{2}\left(1-\frac{1}{2} k s\right)$ \\
\hline Risk sharing (SH) & $\frac{2}{3} \frac{A}{k s}$ & $\frac{x^{\circ}}{2} \frac{v_{1}}{v_{2}}$ & $\frac{1}{18} \frac{x^{\circ}}{L} \frac{v_{1}^{2}}{v_{2}} \alpha \frac{A^{2}}{k s}$ \\
\hline Risk shifting (SFNA) & $\alpha \frac{A}{2}$ & $\frac{x^{\circ}}{2} \frac{v_{1}}{v_{2}}$ & $\frac{1}{8}\left(r_{1}-\frac{1}{2} r_{2}\right) \frac{v_{1}^{2}}{v_{2}} \frac{x^{\circ}}{L} \alpha A^{2}\left(1-\frac{1}{2} k s\right)$ \\
\hline Risk shifting (SFAN) & $\alpha \frac{A}{2}$ & $\frac{x^{\circ}}{2} \frac{p_{1}}{p_{2}} \frac{v_{1}}{v_{2}}$ & $\frac{1}{8} \frac{r_{1}^{2}}{r_{2}} \frac{v_{1}^{2}}{v_{2}} \frac{x^{\circ}}{L} \alpha A^{2}\left(1-\frac{1}{2} k s\right)$ \\
\hline Risk sharing (SHNA) & $\frac{2}{3} \frac{A}{k s}$ & $\frac{x^{\circ}}{2} \frac{v_{1}}{v_{2}}$ & $\frac{1}{18}\left(2 k_{1}-k_{2}\right) \frac{v_{1}^{2}}{v_{2}} \frac{x^{\circ}}{L} \alpha \frac{A^{2}}{k s}$ \\
\hline Risk sharing (SHAN) & $\frac{2}{3} \frac{A}{k s}$ & $\frac{x^{\circ}}{2} \frac{p_{1}}{p_{2}} \frac{v_{1}}{v_{2}}$ & $\frac{1}{18} \frac{x^{\circ}}{L} \frac{r_{1}^{2}}{r_{2}} \frac{v_{1}^{2}}{v_{2}} \alpha \frac{A^{2}}{k s}$ \\
\hline
\end{tabular}

Table 1: Optimal choices in the different settings

From a policy point of view, we can first observe that the number of targeted patients is not affected by risk sharing unless the regulator alters the probability of being listed and the pharmaceutical company anticipates this policy and changes its pricing decisions accordingly. In this latter case, given that $\frac{r_{1}}{r_{2}}>1$ by assumption, the number of patients will increase. This result allows to intepret the findings of other models that showed how risk sharing might have increased the number of treatments. Overtreatment (in terms of allowing patients with low probability of success to receive the drug) may occur, but it should be considered a regulatory failure. If the regulator keeps to its strict listing rules the number of patients treated will not be affected. 
It is also interesting to note that the probability of being listed does not affect price which instead simply depends on the listing process (the regulator sets the price by using the level of expected effectiveness) or the penalty system (the industry may set the level of effectiveness for reimbursement purposes).

When the listing process is not affected by the introduction of risk sharing, it is possible to show ${ }^{11}$ that profit for the company is always lower under a risk-sharing agreement. We can in fact rank the expected profit as follows:

$$
E \Pi^{N R}>E \Pi^{S F}>E \Pi^{S H}
$$

If the probability of being listed is not affected by the introduction of risk sharing the regulator is the benefitter in this process. In fact, the number of patients that are going to be treated is the same, but the ex post price (net of the rebate) is lower than in a system where the expected effectiveness is reimbursed.

In other words these mechanisms increase the price of the drug in the negotiation phase, but owing to the rebate mechanism the expected cost for society is lower than without such agreement.

This means that from an economic point of view such a scheme should not be defined risk-sharing but risk-shifting (Towse and Garrison, 2010). The company in fact agrees to receive an expected profit that is lower than without this scheme. Risk sharing schemes we propose are however softer than a pure risk shifting mechanism as the one presented in section 4.1. The expected profits under the agreements we propose are in fact higher. ${ }^{12}$

The company, confronted with an "all or nothing" offer, prefers to reduce its expected profit against the alternative of not being listed and it is for this reason that it accepts the agreements. Some experts argue that risk-sharing is second option for the pharmaceutical industry: if the normal process fails because the

\footnotetext{
${ }^{11}$ See appendix one

12 The proof in this case is trivial: the agreement presented in section 4.1 is a special case of our risk sharing agreement for the case where $D^{R S R}$ is constrained to be $A / 2$
} 
drug is too costly, the company enters a risk-sharing agreement. Our model adds one important element to this discussion: entering such a risk- shifting agreement is equivalent to accepting a reduction in the price of the new drug. The company may prefer this solution for several reasons:

- the price will be based on a declared efficacy which may be higher than the expected value. In the short run this declaration may be used as a message to physicians and competitors on the confidence the company has in the healing properties of the new drug;

- the company may have acquired new evidence on the effectiveness of the new drug or how to better target the patients and it may prefer to risk paying a penalty than receive a lower price.

However, this may not be the end of the story. The probability of being listed is set by the regulator and in some countries the rules for its setting are not even transparent. In this environment is may well be possible that the regulator allows listing with a higher probability. This effect may be anticipated or not by the industry: in both cases it will have an effect on the profit. We can firstly observe that the profit is increasing in $r_{1}$ as shown by the results of the third column of table 1. For a fix level of $r$; we can then define the level of $r_{1}$ for which the expected profit is equal in the two systems. The results are sumarised in table 2:

$\begin{array}{lllll} & \text { Risk Shifting (NA) } & \text { Risk Shifting (AC) } & \text { Risk Sharing (NA) } & \text { Risk Sharing (AC) } \\ r_{1} & \frac{1}{2} r_{2}+\frac{2}{2-k s} & \sqrt{\frac{2 k}{2-k s}} & \frac{9}{8} k s+\frac{1}{2} r_{2} & \frac{3}{2} \sqrt{r_{2} k s} \\ r_{1 / r_{2}}=1 & \frac{1}{2}+\frac{2}{2-k s} & \sqrt{\frac{2}{2-k s}} & \frac{9}{8} k s+\frac{1}{2} & \frac{3}{2} \sqrt{k s}\end{array}$

If we fix $r_{2}=1$; i.e. we assume that the budget has the same impact on the probability of being listed, we can see that for $r_{l}$ sufficiently high the profit under the "risk shifting" and "risk sharing" regimes may be higher than under benchmark. 


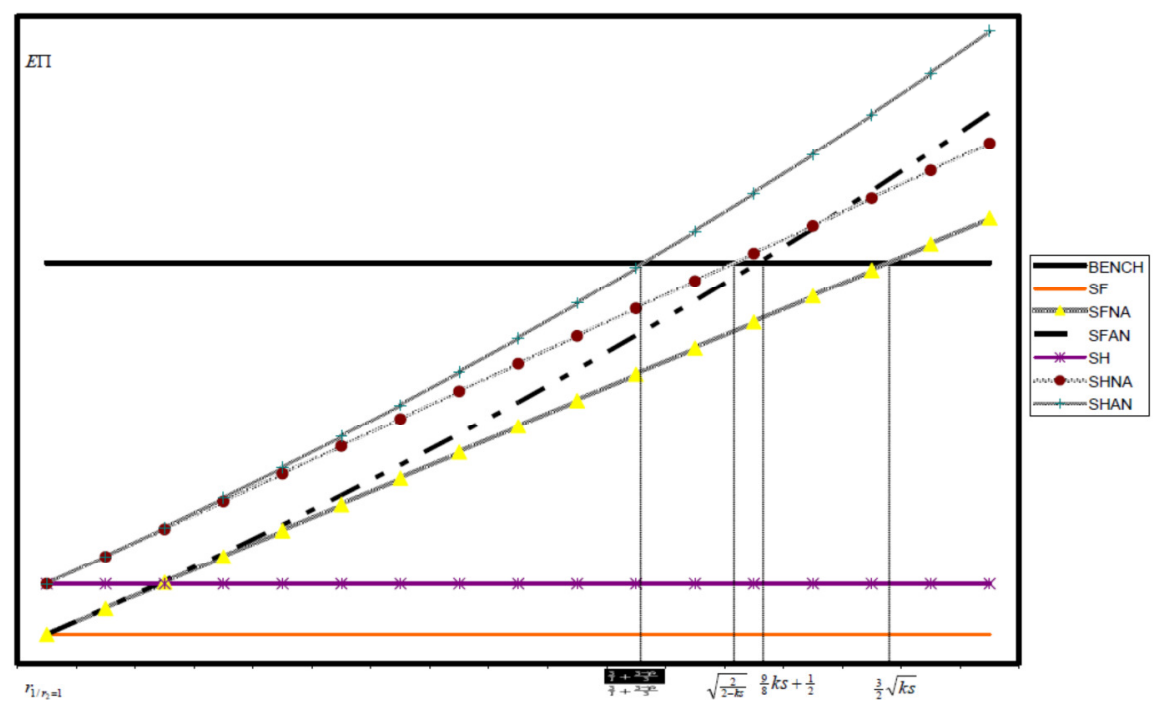

Figure 1: Expected profit under different pricing agreements

The solid line represents the expected profit in the benchmark case. As expected, the profit under "pure" risk sharing and risk shifting schemes are lower than for the benchmark case.

However, if the listing process becomes more generous, the result in terms of expected profit becomes more unpredictable. If the listing process becomes "sufficiently more generous", the industry is better off under risk sharing, inde- pendently of whether it anticipates this change. If it is able to anticipate the change, the expected profit will be however higher.

\section{Conclusions}

In this paper we propose a pricing mechanism to be used both for risk sharing and risk shifting purposes based on the effectiveness of the new drug. 
The price at which the new drug is listed is always higher than in a system without risk sharing, but the expected profit is lower unless the parameters of the listing process are affected by the introduction of risk sharing. This consideration has important policy implications: in the presence of risk sharing the price is not a good proxy for value for money. It is only ex post, when the true effectiveness will be known that value for money can be evaluated. In a risk-sharing agreement in fact it is necessary to take account of the rebates that the firm may incur if the effectiveness falls short of what promised.

At the time of listing, the expected profit of the firm is a better proxy for value for money because it takes account of these rebates. Our formula creates incentives for the company to target the number of people that will be treated instead of increasing it like the risk-sharing mechanism proposed by NICE in 2007 for bortezomib (Barros 2010).

The reason for this different result can be explained as follows: in our approach we use the probability of listing of the new drug and risk sharing on the price while NICE substitutes the listing process with the risk sharing agreement on the price. Our model shows that risk sharing is not a subsititute for total expenditure considerations; on the contrary the two instruments should be used together. The framework we use highlights the effects of the listing process on the number of patients that the company propose to treat in the long run. Our model shows in fact that the number of patients the company proposes to treat depends on the relative weight that is given in the decision process to the efficacy and to budget considerations. This means that all the parameters of the pricing formula have to be carefully assessed to avoid perverse effects (Claxton et al. 2008). For this reason, this model represents a first step in studying new ways of defining prices for drugs in a regulated market. 


\section{References}

[1] Adamsky et al (2010) Risk sharing arrangements for pharmaceuticals: potential considerations and recommendations for European payers, BMC Health Service Research, 10:153

[2] Appleby J, Devlin N, Parkin D, Buxton M, K Chalkidou K. 2009. Searching for cost effectiveness thresholds in the NHS. Health Policy In Press, ISSN 0168-8510, DOI:10.1016/j.healthpol.2008.12.010.

[3] Barros PP (2010) The simple economics of risk-sharing agreements between the NHS and the pharmaceutical industry, Health Economics, forthcoming

[4] Boggild M, Palace J, Barton P et al. Multiple sclerosis risk sharing scheme: two year results of clinical cohort study with historical comparator. BMJ 2009;339:b4677

[5] Capri, S. and R. Levaggi (2006) International Price Regulations in the Pharmaceutical Sector: A Common Model to Sharing the Benefits" Journal of Pharmaceutical Finance, Economics and Policy, 15(2), 21-30

[6] Capri S and Levaggi R (2002) Pricing policies in the pharmaceutical sector. Drug Information Journal 36:453-464.

[7] Claxton K, Briggs A, Buxton MJ, Culyer AJ, McCabe C, Walker S and Sculpher MJ (2008). Value based pricing for NHS drugs: an opportunity not to be missed? British Medical Journal 336;251-254.

[8] Claxton K. (2007) OFT,VBP:QED? Health Economics, 16:545-58.

[9] Cook, J., and Vernon, J., and Manning, R. (2008) "Pharmaceutical Risk Sharing Agreements. "PharmacoEconomics;26(7):551-556

[10] Danzon PM, Towse A (2002) The Economics of Gene Therapy and of Pharmacogenetics. Value in Health 5;1:5-13. 
[11] Danzon PM, Chao LW (2000) Does regulation drive out competition in pharmaceutical markets? Journal of Law and Economics 43, 311-357.

[12] DePouvourville, G. (2006) Risk sharing agreement for innovative drugs, European Journal of Health Economics, 7:155-157 B. Brunekreef, S.T. Holgate (2002), " Air Pollution and Health", Lancet, 360: 1233--1242.

[13] Grifffin, S. K. Claxton, S. Palmer, M. Sculpher (2010)Dangerous omissions: the consequences of ignoring decision uncertainty, Health Economics, DOI: 10.1002/hec. 1586

[14] KCE 2008. Threshold values for cost-effectiveness in health care, KCE reports 100C, Bruxelles

[15] McCabe C, Claxton K, Culyer AJ. 2008. The NICE CostEffectiveness Threshold: What it is and What that Means. PharmacoEconomics 26(9):733-744.

[16] NICE (2007a) Final Appraisal Determination, Bortezomib monotherapy for relapsed multiple myeloma.

[17] NICE (2007) Bortezomib monotherapy for relapsed multiple myeloma. Technology Appraisal Guidance No 129

[18] OECD Health Data 2009: Statistics and Indicators for 30 Countries, 2009.

[19] Pirmohamed M and Lewis G (2004) The Implications of Pharmacogenetics and Pharmacogenomics for Drug Development and Health Care in Mossialos, E. et al (eds), Regulating Pharmaceuticals in Europe: Striving For Efficiency, Equity and Quality (Open University Press, Maidenhead)

[20] Towse A and Garrison LP (2010) Can't Get No Satisfaction? Will Pay for Performance Help? Toward an Economic Framework for Understanding Performance-Based Risk-Sharing Agreements for Innovative Medical Products, Pharmacoeconomics, 28(2): 93-102. 
[21] Zaric GS and O'Brien BJ (2005) Analysis of a pharmaceutical risk sharing agreement based on the purchaser's total budget. Health Economics 14: 793-803.

[22] Zaric GS and Xie B. (2009) The impact of two Pharmaceutical Risk-Sharing agreements on Pricing, Promotion and Net Health Benefits, Value in Health, 12(5), 838-845

[23] Zweifel P. Breyer F. and M.Kifmann (2009) Health Economics, 2nd Edition, Springer, Berlin

[24] http://ec.europa.eu/pharmaforum/docs/pricing_risk_en.pdf 


\section{Appendix A}

In its most general form the maximisation problem can be written as:

$$
\begin{aligned}
\operatorname{Max}_{D, x} E \Pi= & \left(r_{1} v_{1}\left(\frac{D}{D^{\circ}}\right)-r_{2} v_{2}\left(\frac{\alpha D x}{p^{\circ} x^{\circ}}\right)\right)\left(\alpha D\left(1-k \frac{s D}{A}\right)\right) x \\
D \leq & \text { s.t }
\end{aligned}
$$

We can take account of the constraint using Khun Tucker conditions: If the marginal probability of being listed is linear, the F.O.C. can be written as:

$$
\begin{aligned}
& \frac{\partial}{\partial D}:\left(-r_{1} v_{1} p^{\circ} x^{\circ}+r_{2} v_{2} \alpha x D^{\circ}\right) \alpha D x \frac{-2 A+3 k s D}{D^{\circ} p^{\circ} x^{\circ} A} \geq 0 \\
& \frac{\partial}{\partial x}: D^{2} \alpha(-A+k s D) \frac{2 r_{2} v_{2} \alpha x D^{\circ}-r_{1} v_{1} p^{\circ} x^{\circ}}{D^{\circ} p^{\circ} x^{\circ} A} \geq 0 \\
& D \leq A
\end{aligned}
$$

\section{Appendix A.1: Solution for the problem without risk sharing.}

In this case, $k=s=0 ; D=A / 2, r_{1}=r_{2}=1$. The optimal number of patients can be found using equation (17b) which becomes:

$\frac{1}{4} A^{2} \alpha\left(\frac{v_{1}}{D^{\circ}}-2 v_{2} \alpha \frac{x}{p^{\circ} x^{\circ}}\right)=0$

hence:

$$
x^{N R}=\frac{1}{2} v_{1} p^{\circ} \frac{x^{\circ}}{v_{2} \alpha D^{\circ}}=\frac{x^{\circ}}{2} \frac{v_{1}}{v_{2}}
$$

Substituting the optimal quantity in the expected profit function we can find $E \pi^{N R}$

\section{Appendix A.2: Solution for the risk shifting problem}

Appendix A.2.1: Probability of listing equal to $v 1$ and $v 2$

As in the previous case, $D=A / 2 ; r_{1}=r_{2}=1$ while $0 \leq k \leq 1$ and $0 \leq s \leq 1$.

The optimal number of patients can be found using equation ((17b)) which becomes:

$$
\frac{1}{4} A^{2} \alpha\left(\frac{v_{1}}{D^{\circ}}-2 \pi_{2} \alpha \frac{x}{p^{\circ} x^{\circ}}\right)=0
$$


hence:

$$
x^{S F}=\frac{1}{2} v_{1} p^{\circ} \frac{x^{\circ}}{v_{2} \alpha D^{\circ}}=\frac{x^{\circ}}{2} \frac{v_{1}}{v_{2}}
$$

Appendix A.2.2: Probability of listing equal to $r_{1} v_{1}$ and $r_{2} v_{2}$

If the introduction of risk shifting makes the regulator change the probability of listing, the industry may anticipate this change or not.

Change not anticipated. The optimal quantity is still represented by $x^{S F}=\frac{x^{\circ}}{2} \frac{v_{1}}{v_{2}}$,; but in the profit function the probabililty of being listed is represented by $r_{1} v_{1}$ and $r_{2} v_{2}$ hence the expected profit will be equal to $E \Pi^{S F_{N A}}=\frac{1}{8}\left(r_{1}-\frac{1}{2} r_{2}\right) \frac{x^{0}}{L} \frac{v_{1}^{2}}{v_{2}} \alpha A^{2}\left(1-\frac{1}{2} k s\right)$

Anticipated change. In this case, the FOC can be written as:

$$
\frac{1}{4} A^{2} \alpha\left(\frac{r_{1} v_{1}}{D^{\circ}}-2 r_{2} v_{2} \alpha \frac{x}{p^{\circ} x^{\circ}}\right)=0
$$

hence:

$$
x^{S F_{A C}}=\frac{1}{2} r_{1} v_{1} p^{\circ} \frac{x^{\circ}}{r_{2} v_{2} \alpha D^{\circ}}=\frac{x^{\circ}}{2} \frac{r_{1} v_{1}}{r_{2} v_{2}}
$$

Substituting the optimal quantity in the expected profit function we can find $E \pi^{S H A N}$

\section{Appendix A.3: Solution for the risk sharing problem}

Appendix A.3.1: Probability of listing equal to $v_{1}$ and $v_{2}$

In this case $r_{1}=r_{2}=1$. Let's first assume that the constraint is not binding. Equations (17a)and (17b) can be solved as a system of linear equations. The system has multiple solutions, but only the following one is feasible:

$$
\begin{aligned}
& D^{S H}=\frac{2}{3} \frac{A}{k s} \\
& x^{S H}=\frac{1}{2} v_{1} p_{0} \frac{x_{0}}{v_{2} \alpha D_{0}}
\end{aligned}
$$

For $k s<\frac{2}{3}$ the constraint is binding and the problem can be written as: 
$\operatorname{Max}_{x} E \Pi=\left(v_{1}\left(\frac{A}{D^{\circ}}\right)-v_{2}\left(\frac{\alpha A x}{p^{\circ} x^{\circ}}\right)\right)\left(\alpha A(1-k) \int_{0}^{s A} \frac{1}{A} d E\right) x$

The FOC can be written as:

$-A^{2} \alpha(-1+k s) \frac{-2 v_{2} x+v_{1} x^{\circ}}{D^{\circ} x^{\circ}}$

which implies:

$$
x^{S H}=\frac{x^{\circ}}{2} \frac{v_{1}}{v_{2}}
$$

Appendix A.3.2: Probability of listing equal to r1v1 and $r 2 v 2$

If the introduction of risk shifting makes the regulator change the probability of listing, the industry may anticipate this change or not.

Change not anticipated. The optimal quantity is still represented by $D^{S H}=\frac{2}{3} \frac{A}{k s}$ and $x^{S H}=\frac{x^{\circ}}{2} \frac{v_{1}}{v_{2}}$; ; but in the profit function the probability of being listed is represented by $r_{1} v_{1}$ and $r_{2} v_{2}$ hence the expected profit will be equal to

$E \Pi^{S H_{N A}}=\frac{1}{27}\left(2 r_{1}-r_{2}\right) \frac{A^{2}}{k^{2} s^{2} D^{\circ}} \alpha \frac{v_{1}^{2}}{v_{2}} x^{\circ}$

Anticipated change In this case, the FOC can be written as:

$$
\begin{gathered}
\frac{\partial}{\partial D}:\left(-r_{1} v_{1} p^{\circ} x^{\circ}+r_{2} v_{2} \alpha x D^{\circ}\right) \alpha D x \frac{-2 A+3 k s D}{D^{\circ} p^{\circ} x^{\circ} A} \geq 0 \\
\frac{\partial}{\partial x}: D^{2} \alpha(-A+k s D) \frac{2 r_{2} v_{2} \alpha x D^{\circ}-r_{1} v_{1} p^{\circ} x^{\circ}}{D^{\circ} p^{\circ} x^{\circ} A} \geq 0 \\
D \leq A
\end{gathered}
$$

The system has multiple solutions, but only the following one is feasible:

$$
\begin{aligned}
& D^{S H_{A C}}=\frac{2}{3} \frac{A}{k s} \\
& x^{S H_{A C}}=\frac{1}{2} r_{1} v_{1} p_{0} \frac{x_{0}}{r_{2} v_{2} \alpha D_{0}}
\end{aligned}
$$

For $k s<\frac{2}{3}$ the constraint is binding and the problem can be written as: 
$\operatorname{Max}_{x} E \Pi=\left(r_{1} v_{1}\left(\frac{A}{D^{\circ}}\right)-r_{2} v_{2}\left(\frac{\alpha A x}{p^{\circ} x^{\circ}}\right)\right)\left(\alpha A(1-k) \int_{0}^{s A} \frac{1}{A} d E\right) x$

The FOC can be written as:

$$
-A^{2} \alpha(-1+k s) \frac{-2 r_{2} v_{2} x+r_{1} v_{1} x^{\circ}}{D^{\circ} x^{\circ}}
$$

which implies:

$$
x^{S H_{A N}}=\frac{x^{\circ}}{2} \frac{r_{1} v_{1}}{r_{2} v_{2}}
$$


Working Papers recently published

(The complete list of working papers can be found at http.//www.disefin.unige.it)

n.1/2011 Cinzia Di Novi, "The Indirect Effect of Fine Particulate Matter on Health through Individuals' Life-style"

n.4/2010 Angelo Baglioni, Andrea Monticini, "Why does the Interest Rate Decline Over the Day? Evidence from the Liquidity Crisis"

n.3/2010 Amedeo Fossati: "The double taxation of savings: the Italian debate revisited"

n.2/2010 Andrea Monticini, David Peel, Giacomo Vaciago: "The impact of ECB and FED announcements on the Euro Interest Rates"

n.1/2010 Amedeo Fossati: "Vilfredo Pareto and the methodology of the Italian tradition in public finance" 